\title{
123.
}

\section{ON THE GEOMETRICAL REPRESENTATION OF AN ABELIAN INTEGRAL.}

[From the Philosophical Magazine, vol. vi. (1853), pp. 414-418.]

THE equation of a surface passing through the curve of intersection of the surfaces

$$
\begin{array}{r}
x^{2}+y^{2}+z^{2}+w^{2}=0, \\
a x^{2}+b y^{2}+c z^{2}+d w^{2}=0
\end{array}
$$

is of the form

$$
\curlyvee\left(x^{2}+y^{2}+z^{2}+w^{2}\right)+a x^{2}+b y^{2}+c z^{2}+d w^{2}=0,
$$

where $\gamma$ is an arbitrary parameter. Suppose that the surface touches a given plane, we have for the determination of $\gamma$ a cubic equation the roots of which may be considered as parameters defining the plane in question. Let one of the values of $\gamma$ be considered equal to a given quantity $k$, the plane touches the surface

$$
k\left(x^{2}+y^{2}+z^{2}+w^{2}\right)+a x^{2}+b y^{2}+c z^{2}+d w^{2}=0,
$$

and the other two values of $\varangle$ may be considered as parameters defining the particular tangent plane, or what is the same thing, determining its point of contact with the surface. surface

Or more clearly, thus:-in order to determine the position of a point on the

$$
k\left(x^{2}+y^{2}+z^{2}+w^{2}\right)+a x^{2}+b y^{2}+c z^{2}+d w^{2}=0 ;
$$

the tangent plane at the point in question is touched by two other surfaces

$$
\begin{aligned}
& p\left(x^{2}+y^{2}+z^{2}+w^{2}\right)+a x^{2}+b y^{2}+c z^{2}+d w^{2}=0, \\
& q\left(x^{2}+y^{2}+z^{2}+w^{2}\right)+a x^{2}+b y^{2}+c z^{2}+d w^{2}=0 ;
\end{aligned}
$$

C. II. 
and, this being so, $p$ and $q$ are the parameters by which the point in question is determined. We may for shortness speak of the surface

$$
k\left(x^{2}+y^{2}+z^{2}+w^{2}\right)+a x^{2}+b y^{2}+c z^{2}+d w^{2}=0
$$

as the surface $(k)$. It is clear that we shall then have to speak of

$$
x^{2}+y^{2}+z^{2}+w^{2}=0
$$

as the surface $(\infty)$.

I consider now a chord of the surface $(\infty)$ touching the two surfaces $(k)$ and $\left(k^{\prime}\right)$; and I take $\theta, \phi$ as the parameters of the one extremity of this chord; $(p, q)$ as the parameters of the point of contact with the surface $(k) ; p^{\prime}, q^{\prime}$ as the parameters of the point of contact with the surface $\left(k^{\prime}\right)$; and $\theta^{\prime}, \phi^{\prime}$ as the parameters of the other extremity of the chord; the points in question may therefore be distinguished as the points $(\infty ; \theta, \phi),(k ; p, q),\left(k^{\prime} ; p^{\prime}, q^{\prime}\right)$, and $\left(\infty ; \theta^{\prime}, \phi^{\prime}\right)$. The caordinates of the point $(\infty ; \theta, \phi)$ are given by

$$
\begin{aligned}
x: y: z: w= & \sqrt{(a+\theta)(a+\phi)} \div \sqrt{(a-b)(a-c)(a-d)} \\
& : \sqrt{(b+\theta)(b+\phi)} \div \sqrt{(b-c)(b-d)(b-a)} \\
& : \sqrt{(c+\theta)(c+\phi)} \div \sqrt{(c-d)(c-a)(c-b)} \\
& : \sqrt{(d+\theta)(d+\phi)} \div \sqrt{(d-a)(d-b)(d-c)}
\end{aligned}
$$

those of the point $(k ; p, q)$ by

$$
\begin{aligned}
x: y: z: w= & \sqrt{(a+p)(a+q)} \div \sqrt{(a-b)(a-c)(a-d)} \sqrt{a+k} \\
& : \sqrt{(b+p)(b+q)} \div \sqrt{(b-c)(b-d)(b-a)} \sqrt{b+k} \\
& : \sqrt{(c+p)(c+q)} \div \sqrt{(c-d)(c-a)(c-b)} \sqrt{c+k} \\
& : \sqrt{(d+p)(d+q)} \div \sqrt{(d-a)(d-b)(d-c)} \sqrt{d+k}
\end{aligned}
$$

and similarly for the other two points.

Consider, in the first place, the chord in question as a tangent to the two surfaces $(k)$ and $\left(k^{\prime}\right)$. It is clear that the tangent plane to the surface $(k)$ at the point $(k ; p, q)$ must contain the point $\left(k^{\prime} ; p^{\prime}, q^{\prime}\right)$, and vice versâ. Take for a moment $\xi, \eta, \zeta, \omega$ as the coordinates of the point $(k ; p, q)$, the equation of the tangent plane to $(k)$ at this point is

$$
\Sigma(a+k) \xi x=0
$$

or substituting for $\xi, \ldots$ their values

$$
\Sigma(x \sqrt{(a+p)(a+q)} \sqrt{a+k} \div \sqrt{(a-b)(a-c)(a-d)})=0 ;
$$


or taking for $x, \ldots$ the coordinates of the point $\left(k^{\prime}, p^{\prime}, q^{\prime}\right)$, we have for the conditions that this point may lie in the tangent plane in question,

$$
\Sigma\left(\sqrt{(a+p)(a+q)} \sqrt{\left(a+p^{\prime}\right)\left(a+q^{\prime}\right)} \sqrt{(a+k)} \div \sqrt{\left(a+k^{\prime}\right)}(a-b)(a-c)(a-d)\right)=0 ;
$$

or under a somewhat more convenient form we have

$$
\Sigma\left((b-c)(c-d)(d-b) \sqrt{(a+p)(a+q)} \sqrt{\left(a+p^{\prime}\right)\left(a+q^{\prime}\right)} \frac{\sqrt{a+k}}{\sqrt{a+k^{\prime}}}\right)=0,
$$

for the condition in order that the point $\left(k^{\prime}, p^{\prime}, q^{\prime}\right)$ may lie in the tangent plane at $(k ; p, q)$ to the surface $(k)$. Similarly, we have

$$
\Sigma\left((b-c)(c-d)(d-b) \sqrt{(a+p)(a+q)} \sqrt{\left(a+p^{\prime}\right)\left(a+q^{\prime}\right)} \frac{\sqrt{a+k^{\prime}}}{\sqrt{a+k}}\right)=0,
$$

for the condition in order that the point $(k, p, q)$ may lie in the tangent plane at $\left(k^{\prime} ; p^{\prime}, q^{\prime}\right)$ to the surface $\left(k^{\prime}\right)$. The former of these two equations is equivalent to the system of equations

$$
\sqrt{(a+p)(a+q)\left(a+p^{\prime}\right)\left(a+q^{\prime}\right)} \quad \sqrt{\frac{a+k}{a+k^{\prime}}}=\lambda+\mu a+\nu a^{2},
$$

and the latter to the system of equations

$$
\left.\sqrt{(a+p)(a+q)\left(a+p^{\prime}\right)\left(a+q^{\prime}\right.}\right) \sqrt{\frac{a+k^{\prime}}{a+k}}=\lambda^{\prime}+\mu^{\prime} a+\nu^{\prime} a^{2} ;
$$

where in each system $a$ is to be successively replaced by $b, c, d$, and where $\lambda, \mu, \nu$ and $\lambda^{\prime}, \mu^{\prime}, \nu^{\prime}$ are indeterminate. Now dividing each equation of the one system by the corresponding equation in the other system, we see that the equation

$$
\frac{x+k}{x+k^{\prime}}=\frac{\lambda+\mu x+\nu x^{2}}{\lambda^{\prime}+\mu^{\prime} x+\nu^{\prime} x^{2}}
$$

is satisfied by the values $a, b, c, d$ of $x$; and, therefore, since the equation in $x$ is only of the third order, that the equation in question must be identically true. We may therefore write

$$
\lambda+\mu x+\nu x^{2},=(\rho x+\sigma)(x+k), \quad \lambda^{\prime}+\mu^{\prime} x+\nu^{\prime} x^{2}=(\rho x+\sigma)\left(x+k^{\prime}\right),
$$

and the two systems of equations become therefore equivalent to the single system,

$$
\begin{aligned}
& \sqrt{(a+p)(a+q)\left(a+p^{\prime}\right)\left(a+q^{\prime}\right)}=(\rho a+\sigma) \sqrt{(a+k)\left(a+k^{\prime}\right),} \\
& \sqrt{(b+p)(b+q)\left(b+p^{\prime}\right)\left(b+q^{\prime}\right)}=(\rho b+\sigma) \sqrt{(b+k)\left(b+k^{\prime}\right)}, \\
& \sqrt{(c+p)(c+q)\left(c+p^{\prime}\right)\left(c+q^{\prime}\right)}=(\rho c+\sigma) \sqrt{(c+k)\left(c+k^{\prime}\right)}, \\
& \sqrt{(d+p)(d+q)\left(d+p^{\prime}\right)\left(d+q^{\prime}\right)}=(\rho d+\sigma) \sqrt{(d+k)\left(d+k^{\prime}\right)},
\end{aligned}
$$


a set of equations which may be represented by the single equation

$$
\psi(x+p)(x+q)\left(x+p^{\prime}\right)\left(x+q^{\prime}\right)-(\rho x+\sigma)^{2}(x+k)\left(x+k^{\prime}\right)=\chi(x-a)(x-b)(x-c)(x-d),
$$

where $x$ is arbitrary; or what is the same thing, writing $-x$ instead of $x$,

$$
\chi(x+a)(x+b)(x+c)(x+d)+(\rho x-\sigma)^{2}(x-k)\left(x-k^{\prime}\right)=\psi(x-p)(x-q)\left(x-p^{\prime}\right)\left(x-q^{\prime}\right) .
$$

Hence, putting

$$
\begin{aligned}
& \Pi x=\int \frac{d x}{\sqrt{(x+a)(x+b)(x+c)(x+d)(x-k)\left(x-k^{\prime}\right)}}, \\
& \Pi x=\int \frac{x d x}{\sqrt{(x+a)(x+b)(x+c)(x+d)(x-k)\left(x-k^{\prime}\right)}},
\end{aligned}
$$

we see that the algebraical equations between $p, q ; p^{\prime}, q^{\prime}$ are equivalent to the transcendental equations

$$
\begin{aligned}
& \Pi p \pm \Pi q \pm \Pi p^{\prime} \pm \Pi q^{\prime}=\text { const. } \\
& \Pi_{\iota} p \pm \Pi, q \pm \Pi, p^{\prime} \pm \Pi, q^{\prime}=\text { const. }
\end{aligned}
$$

The algebraical equations which connect $\theta, \phi$ with $p, q ; p^{\prime}, q^{\prime}$, may be exhibited under several different forms; thus, for instance, considering the point $(\infty ; \theta, \phi)$ as a point in the line joining $(k ; p, q)$ and $\left(k^{\prime} ; p^{\prime}, q^{\prime}\right)$, we must have

$$
\left\|\begin{array}{ll}
\sqrt{(a+p)(a+q)} \div \sqrt{a+k}, & \sqrt{(b+p)(b+q)} \div \sqrt{b+k}, \ldots \\
\sqrt{\left(a+p^{\prime}\right)\left(a+q^{\prime}\right)} \div \sqrt{a+k^{\prime}}, & \sqrt{\left(b+p^{\prime}\right)\left(b+q^{\prime}\right)} \div \sqrt{b+k^{\prime}} \\
\sqrt{(a+\theta)(a+\phi)}, & \sqrt{(b+\theta)(b+\phi)}
\end{array}\right\|=0,
$$

i.e. the determinants formed by selecting any three of the four columns must vanish; the equations so obtained are equivalent (as they should be) to two independent equations.

Or, again, by considering $(\infty ; \theta, \phi)$ first as a point in the tangent plane at $(k ; p, q)$ to the surface $(k)$, and then as a point in the tangent plane at $\left(k^{\prime} ; p^{\prime}, q^{\prime}\right)$ to the surface $\left(k^{\prime}\right)$, we obtain

$$
\begin{aligned}
& \Sigma((b-c)(c-d)(d-b) \sqrt{(a+p)(a+q)} \sqrt{(a+k)} \sqrt{(a+\theta)(a+\phi)})=0, \\
& \Sigma\left((b-c)(c-d)(d-b) \sqrt{\left(a+p^{\prime}\right)\left(a+q^{\prime}\right)} \sqrt{\left(a+k^{\prime}\right)} \sqrt{(a+\theta)(a+\phi)}\right)=0 .
\end{aligned}
$$

Or, again, we may consider the line joining $(\infty ; \theta, \phi)$ and $(k ; p, q)$ or $\left(k^{\prime} ; p^{\prime}, q^{\prime}\right)$, as touching the surfaces $(k)$ and $\left(k^{\prime}\right)$; the formulæ for this purpose are readily obtained by means of the lemma,- 
"The condition in order that the line joining the points $(\xi, \eta, \zeta, \omega)$ and $\left(\xi^{\prime}, \eta^{\prime}, \zeta^{\prime}, \omega^{\prime}\right)$ may touch the surface

$$
\mathrm{a} x^{2}+\mathrm{b} y^{2}+\mathrm{c} z^{2}+\mathrm{d} w^{2}=0
$$

is

$$
\Sigma a b\left(\xi \eta^{\prime}-\xi^{\prime} \eta\right)^{2}=0,
$$

the summation extending to the binary combinations of a, b, c, d."

But none of all these formulæ appear readily to conduct to the transcendental equations connecting $\theta, \phi$ with $p, q ; p^{\prime}, q^{\prime}$. Reasoning from analogy, it would seem that there exist transcendental equations

$$
\begin{aligned}
& \pm \Pi \theta \pm \Pi \phi \pm \Pi p \pm \Pi p^{\prime}=\text { const. } \\
& \pm \Pi, \theta \pm \Pi, \phi \pm \Pi, p \pm \Pi, p^{\prime}=\text { const. }
\end{aligned}
$$

or the similar equations containing $q, q^{\prime}$, instead of $p, p^{\prime}$, into which these are changed by means of the transcendental equations between $p, q, p^{\prime}, q^{\prime}$. If in these equations we write $\theta^{\prime}, \phi^{\prime}$ instead of $\theta, \phi$, it would appear that the functions $\Pi p, \Pi p^{\prime}, \Pi_{1} p, \Pi_{l} p^{\prime}$ may be eliminated, and that we should obtain equations such as

$$
\begin{aligned}
& \pm \Pi \theta \pm \Pi \phi \pm \Pi \theta^{\prime} \pm \Pi \phi^{\prime}=\text { const. } \\
& \pm \Pi, \theta \pm \Pi, \phi \pm \Pi \theta^{\prime} \pm \Pi, \phi^{\prime}=\text { const. }
\end{aligned}
$$

to express the relations that must exist between the parameters $\theta, \phi$ and $\theta^{\prime}, \phi^{\prime}$ of the extremities of a chord of the surface

$$
x^{2}+y^{2}+z^{2}+w^{2}=0,
$$

in order that this chord may touch the two surfaces

$$
\begin{aligned}
& k\left(x^{2}+y^{2}+z^{2}+w^{2}\right)+a x^{2}+b y^{2}+c z^{2}+d w^{2}=0, \\
& k^{\prime}\left(x^{2}+y^{2}+z^{2}+w^{2}\right)+a x^{2}+b y^{2}+c z^{2}+d w^{2}=0 .
\end{aligned}
$$

The quantities $k, k^{\prime}$, it will be noticed, enter into the radical of the integrals $\Pi x, \Pi x$. This is a very striking difference between the present theory and the analogous theory relating to conics, and leads, I think, to the inference that the theory of the polygon inscribed in a conic, and the sides of which touch conics intersecting the conic in the same four points, cannot be extended to surfaces in such manner as one might be led to suppose from the extension to surfaces of the much simpler theory of the polygon inscribed in a conic, and the sides of which touch conics having double contact with the conic. (See my paper "On the Homographic Transformation of a surface of the second order into itself," [122]).

The preceding investigations are obviously very incomplete; but the connexion which they point out between the geometrical question and the Abelian integral involving the root of a function of the sixth order, may I think be of service in the theory of these integrals. 